\title{
To overlap or not to overlap: Enabling Channel Bonding in High Density WLANs
}

\author{
Sergio Barrachina-Muñoz, Francesc Wilhelmi, Boris Bellalta
}

\begin{abstract}
Wireless local area networks (WLANs) are the most popular kind of wireless Internet connection. However, the number of devices accessing the Internet through WLANs such as laptops, smartphones, or wearables, is increasing drastically at the same time that applications' throughput requirements do. To cope with the later challenge, channel bonding (CB) techniques are used for enabling higher data rates by transmitting in wider channels. Nonetheless, some important issues such as higher potential co-channel interference arise when bonding channels. In this paper we address this point at issue: is it convenient for high density WLANs to use wider channels and potentially overlap in spectrum? We show that, while the performance of static CB is really poor, spectrum overlapping is highly convenient when adapting to the medium through dynamic channel bonding (DCB); specially for low to moderate traffic loads. Contradicting most of current thoughts, the presented results suggest that future wireless networks should be allowed to use all available spectrum, and locally adapt to desirable configurations.
\end{abstract}

Index Terms-Dynamic channel bonding, WLAN, spatial distribution, traffic load, IEEE 802.11ax

\section{INTRODUCTION}

Even though remarkable technological improvements have been achieved in the last decades, wireless local area networks (WLANs), with IEEE 802.11's Wi-Fi as the most widely used standard, still face important challenges that may degrade their performance. Specifically, frequency spectrum is becoming scarce and inefficient because of the increasing number of wireless devices, the characteristically heterogeneous and random WLAN deployments, and the raising throughput demands (e.g., some virtual reality applications require more than 1 Gbps to operate properly [1]). All these circumstances lead to dense scenarios with coexistence issues where WLANs seek for selfishly serving their users in non-collaborative deployments.

As a result, there is a clear need of exploiting the spectrum in a more efficient way by maximizing transmissions' bandwidth. One of the most promising techniques to overcome such a challenge is channel bonding (CB) [2]. The main idea behind $\mathrm{CB}$ is to allow using wider channels in order to transmit at higher transmission rates and increasing the throughput accordingly. CB for WLANs was firstly introduced in the IEEE $802.11 n-2009$ amendment [3] by letting two separated $20 \mathrm{MHz}$ channels (or basic channels) get combined into a 40 $\mathrm{MHz}$ channel. Later, IEEE 802.11ac-2013 [4] introduced the capability of transmitting in 80 and $160 \mathrm{MHz}$ channels. Future amendments like the IEEE 802.11ax-2019 (11ax) are expected to boost the use of wider channels [5]. A survey of CB schemes for different types of wireless networks is provided in [6].

All the authors are with Universitat Pompeu Fabra (UPF).

E-mails: \{sergio.barrachina, boris.bellalta, francisco.wilhelmi\}@upf.edu
There are important drawbacks, however, when it comes to transmitting in wider channels: essentially, the larger the bandwidth used for transmitting, the higher the co-channel and adjacent channel interference perceived by neighboring nodes. That is, CB may be counterproductive in terms of performance if not properly implemented. In this regard, dynamic channel bonding (DCB) allows adapting the selected transmission bandwidth to the channel status right before transmitting. This provides a higher degree of flexibility that improves the average performance in a simple and efficient way.

Then, we can differentiate two approaches with respect to spectrum management in WLANs: $i$ ) fostering transmissions in non-overlapping basic channels, or $\mathrm{ii}$ ) enabling faster transmissions in wider channels that may potentially overlap in spectrum. Alas, in high density (HD) spatially distributed ${ }^{1}$ scenarios, the complex interrelations given among nodes (located inside or outside the carrier sense range of each other) complicate the task of a priori estimating the optimal overlapping approach on a per-WLAN basis.

To the best of our knowledge, even after significant research has been conducted on the impact of DCB on spatially distributed WLANs' performance under saturation regimes, the effects of unsaturated patterns are still unknown. While saturated regimes offer valuable insights on worst case scenarios, WLANs' are characteristically unsaturated with load patterns that deeply depend on the application/s being supported.

In this paper we compare the traditional single-channel and DCB approaches in networks of WLANs under variable traffic loads. By means of simulations, we evaluate the performance of the aforementioned approaches in terms of throughput and delay achieved in HD 11ax [7] WLAN scenarios. Results show that, while the performance of static $\mathrm{CB}$ is clearly poor, spectrum-adapting DCB significantly outperforms the traditional single-channel, being the best approach for future WLANs. Notwithstanding, fairness issues like flow starvations may result as a consequence of implementing aggressive DCB in spatially distributed deployments. So, further research is required in this topic.

\section{DYNAMIC CHANNEL BONDING}

\section{A. Dynamic channel bonding in WLANs}

DCB is a useful mechanism for accommodating growing WLAN data capacity requirements. Essentially, DCB is a technique whereby nodes, i.e., access points (APs) and stations (STAs), are allowed to use contiguous sets of available basic channels for their transmissions, thus potentially achieving

\footnotetext{
${ }^{1}$ In spatially distributed scenarios nodes are not necessary within the carrier sense range of each other.
} 
higher throughput [8], [9]. Namely, by doubling the channel bandwidth, approximately the double data capacity can be achieved.

However, implementing DCB in ever-increasingly complex WLAN networks requires a careful balance of trade-offs. Firstly, regarding channelization, ${ }^{2}$ the density of neighboring nodes and the number of independent basic channels (which are regulated by governmental institutions) determine the feasibility of deploying interference-free networks. Essentially, as transmission channels get wider, frequency spectrum reuse becomes arduous, and the probability of packet collisions due to co-channel and adjacent channel interference increases. Secondly, the higher the bandwidth, the smaller the transmitted power per $\mathrm{Hz}$ and corresponding coverage range. This, on the one hand reduces the interference with other WLANs operating in a (partially) overlapping spectrum. On the other hand, however, it also reduces the SINR, resulting in lower transmission rates.

In this regard, the multiple spatial distribution factors such as transmission powers, clear channel assessment (CCA) levels, transmission channels or environment's path loss, make it really difficult to generalize to an optimal set of rules for transmission channel selection. It follows that bandwidth adaptation is required in order to cope with the challenging scenarios of next-generation WLANs.

\section{B. DCB policies and CSMA/CA operation}

According to the carrier-sense multiple access with collision avoidance (CSMA/CA), when a node $n$ belonging to a WLAN $w$ has a packet ready for transmission, it measures the power sensed in its primary channel $p_{w}$, and determines if it is idle or occupied according to the CCA level. Once $p_{w}$ has been detected idle, $n$ starts the backoff procedure by selecting a random initial value $b \in[0, \mathrm{CW}-1]$, where $\mathrm{CW}$ is the contention window. After computing $b$, the node starts decreasing its counter while sensing the primary channel. Whenever the power sensed by $n$ at $p_{w}$ is higher than its CCA, the backoff is paused until $p_{w}$ is detected free again, at which point the countdown is resumed. When the backoff timer expires, the node selects the transmission channel $C_{n}^{\mathrm{tx}}$ based on the set of idle basic channels ${ }^{3}$ and on the implemented spectrum management rules.

In this paper we refer to such rules as DCB policies. Namely, when the backoff terminates, the node operates according to the DCB policy as follows:

- Static channel bonding (SCB): exclusively picks the whole allocated channel if found free.

- Always-max (AM): picks the widest possible channel found free.

- Probabilistic uniform (PU): picks with same probability any of the possible channels found free.

\footnotetext{
${ }^{2}$ Channelization is the process of setting independent channels on neighboring APs in order to avoid interference among their WLANs.

${ }^{3}$ Note that, in order to include secondary basic channels for transmitting, a WLAN must listen them free during at least a Point coordination function (PCF) Interframe Space (PIFS) period before the backoff counter terminates, as shown in Figure 1.
}

If $\mathrm{CB}$ is not considered, we simply refer to single-channel (SC) operation, i.e., a node can only pick its primary channel for transmitting.

The selected transmission channel is then used throughout the packet exchanges involved in a data packet transmission (i.e., RTS, CTS, data and ACK). Likewise, any other node that receives an RTS in its primary channel with enough power to be decoded will enter in network allocation vector (NAV) state, which is used for deferring channel access and avoiding packet collisions (especially those caused by hidden node situations).

In Figure 1, the temporal evolution of a node operating under the different DCB policies is shown. In this example, the node is allowed to transmit in the set of basic channels $C_{w}=\{1(p), 2,3,4\}$, where $p_{w}=1$ is the primary channel. While SC picks just the primary channel, the rest of policies try to bond channels in different ways. In this regard, SCB is highly inefficient in scenarios with partial interference. In fact, no packets can be transmitted with SCB in this example since the basic channel $\{3\} \in C_{w}$ is busy when the backoff terminates. However, more flexible approaches like AM and PU are able to transmit more than one packet in the same period of time.

On the one hand, AM adapts in an aggressive way to the channel state. Specifically, it is able to transmit in 40 and $80 \mathrm{MHz}$ channels at the end of the first and second backoff, respectively. On the other hand, the stochastic nature of PU makes it more conservative than AM. In the example, the node could transmit in 1 or 2 basic channels with same probability (1/2). Likewise, after the second backoff, a channel composed of 1,2 or 4 basic channels could be selected with probability $(1 / 3)$.

\section{UNDERSTANDING THE INTERACTIONS BETWEEN SPATIALLY DISTRIBUTED WLANS}

In this Section we analytically study the interactions given in spatially distributed WLANs under variable traffic load. We also present two toy scenarios where overlapping approaches are beneficial.

\section{A. The CTMN model for WLANs}

Continuous time Markov networks (CTMNs) have been widely used to model the behavior of WLAN networks. An approach which accurately models the behavior of nonsaturated CSMA/CA networks operating in CS was introduced in [10]. Such model is extended in [11] to capture the coupled dynamics of a group of overlapping WLANs using CB. Later, authors in [12] introduced a framework (SFCTMN) which extended the CTMN algorithm presented in [13] for characterizing DCB policies in spatially distributed scenarios. However, to the best of our knowledge, spatial distribution effects like WLAN starvation are not considered in works studying DCB WLANs under non-saturated regimes.

Below we describe such scenarios by modeling them also through CTMNs. For simplicity, we consider only downlink traffic and that each WLAN is composed by an AP and a single STA. Hence, we simply refer to the WLAN activity as a single entity. 


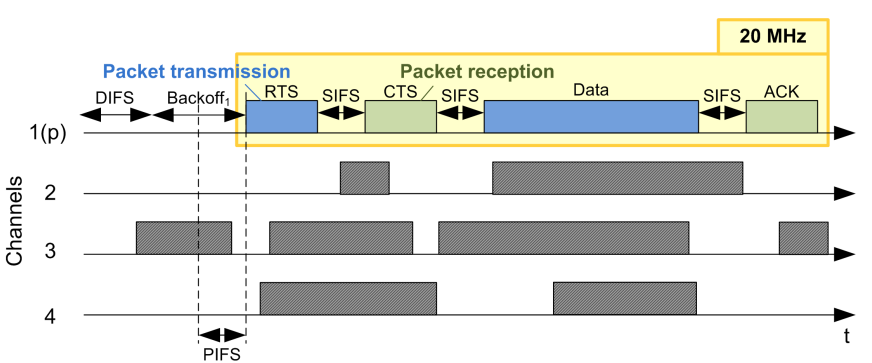

(a) SC

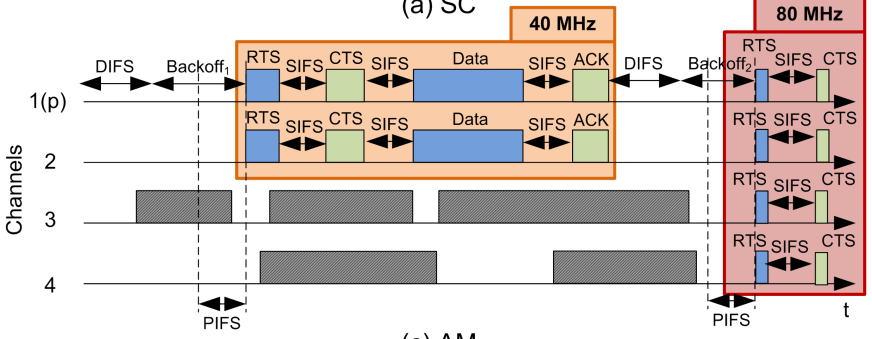

(c) AM

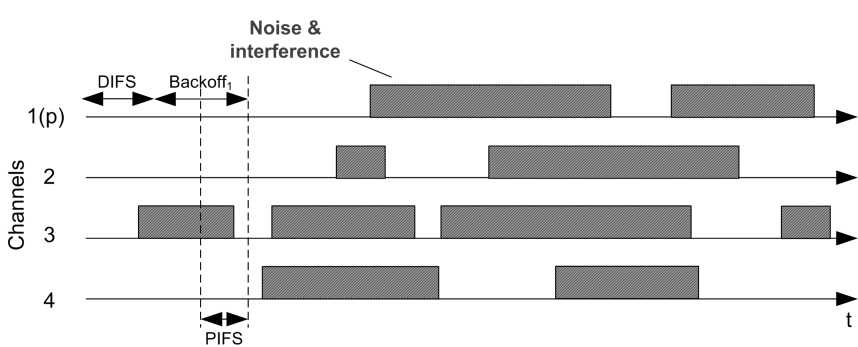

(b) SCB

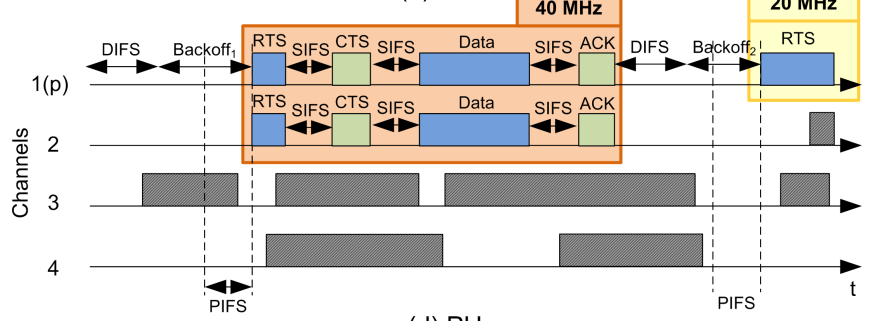

(d) PU

Fig. 1: CSMA/CA temporal evolution of a node operating under different DCB policies in a 11ax channelization scheme.

1) Assumptions and implications: Modeling WLAN scenarios with CTMNs requires the backoff and transmission times to be exponentially distributed. We also assume that the propagation delay between any two pair of nodes is negligible. This has a main implication: the probability of packet collisions between two or more nodes within the carrier sense range of the other nodes is zero. Besides, an infinite maximum number of retransmissions per packet is assumed. ${ }^{4}$

2) States in the CTMN: A state $s$ in the CTMN is defined by the set of WLANs active and the basic channels on which they are transmitting. The set of feasible states is represented by $\mathcal{S}$. Essentially, with slight abuse of notation, we say that a WLAN $w$ is active in state $s$, i.e., $w \in s$ if it is transmitting, and inactive otherwise. States are represented by the most left and most right basic channels used in the transmission channels of each of the active WLANs. For instance, in state $s=\mathrm{A}_{2}^{2} \mathrm{~B}_{1}^{4}$, there are two active WLANs: $\mathrm{A}$ and $\mathrm{B}$. While $\mathrm{A}$ is transmitting in the basic channel $C_{\mathrm{A}}^{\mathrm{tx}}=\{2\}(20 \mathrm{MHz}), \mathrm{B}$ is doing so in a bonded channel $C_{\mathrm{B}}^{\mathrm{tx}}=\{1,2,3,4\}(80 \mathrm{MHz})$. The state in which there is no active WLAN is represented by $\emptyset$.

A transition between two states $s$ and $s^{\prime}$ in the CTMN has a corresponding transition rate $Q_{s, s^{\prime}}$. For forward transitions, the average packet transmission attempt rate is $\rho_{w} \lambda_{w}$, where $\lambda=1 /\left(\mathrm{E}[B] \cdot T_{\text {slot }}\right)$, being $\mathrm{E}[B]$ the expected backoff duration in time slots. Parameter $\rho_{w}$ is the long-run stationary probability that WLAN $w$ has packets ready for transmission when the primary channel is sensed idle and so the backoff counter is active. For backward transitions, the departure rate $(\mu)$ depends on the duration of a successful transmission $\left(T_{\text {suc }}\right)$, which in turn depends on both the data rate $(r)$ given by the selected modulation coding scheme (MCS) and transmission channel width, and on the average data packet length $(\mathrm{E}[L])$. Thus, we simply say that the data rate of a WLAN $w$ depends on the state of the system, which contains such information, i.e.,

\footnotetext{
${ }^{4}$ The effect of assuming infinite maximum number of retransmissions is almost negligible in most of the cases because of the small probability of retransmitting a data packet more than a few times [14].
}

$\mu_{w}(s)$.

3) Performance metrics: The equilibrium distribution vector $\vec{\pi}$ represents the fraction of time the system spends in each state. We define $\pi_{s}$ as the probability of finding the system at state $s$. Hereof, in continuous-time Markov processes with stationary distribution, $\vec{\pi}$ is given by solving the system of equations $Q \pi=0$, where the matrix item $Q_{s, s^{\prime}}$ is the transition rate from state $s$ to $s^{\prime}$. Once $\vec{\pi}$ is computed, estimating the average throughput experienced by each WLAN is straightforward. Specifically, the average throughput of WLAN $w$ is

$$
\Gamma_{w}:=\mathrm{E}[L]\left(\sum_{s \in \mathcal{S}}\left\{\gamma_{w}(s)>\mathrm{CE}: 0,1\right\} \mu_{w}(s) \pi_{s}(1-\eta)\right),
$$

where $\mathrm{E}[L]$ is the expected data packet length, $\gamma_{w}(s)$ is the SINR perceived by the receiving STA in WLAN $w$ in state $s$, $\mathrm{CE}$ is the capture effect threshold, and $\eta$ is the packet error probability.

Note that the unknown $\rho$ parameters must be obtained by solving a non-linear system of equations, which in general does not have a closed-form. As done in [11], in this work we use an iterative fixed-point approach for updating the $\rho$ values until the throughput of all the WLANs converges to their corresponding traffic load, or they become saturated.

\section{B. Toy scenario I: constructing CTMNs for CSMA/CA WLANs}

Let us consider the toy Scenario I shown in Figure 2a, which is composed of two potentially overlapping WLANs. The channel allocation of such scenario can be defined as $C: C_{\mathrm{A}}=\{1(p), 2\}$ with $p_{\mathrm{A}}=1$, and $C_{\mathrm{B}}=\{1,2(p)\}$ with $p_{\mathrm{B}}=2$. That is, there are two basic channels in the system, and the set of valid transmission channels according to the $11 \mathrm{ax}$ channel access scheme is $\{\{1\},\{2\},\{1,2\}\}$. We say that both WLANs are potentially overlapping because they are inside the carrier sense range of each other and thus their signals will overlap when transmitting in the same channel at the same time $t$, i.e., when $C_{\mathrm{A}}^{\mathrm{tx}}(t) \cap C_{\mathrm{B}}^{\mathrm{tx}}(t) \neq \emptyset$. In this case, due to the 
primary channel allocation, A and B will only overlap when both transmit in their whole allocated channel $\{1,2\}$.

Different feasible states and forward transitions may exist in the CTMN depending on the implemented DCB policies. Every feasible transition is weighted by a transition probability vector $\alpha_{w, s}\left(s^{\prime}\right)$ whose elements determine the probability of WLAN $w$ to transit from state $s$ to $s^{\prime}$. Table 2 b collects the number of feasible states $(|\mathcal{S}|)$ and transition probabilities that are given for each of the studied DCB policies in Scenario I. The corresponding CTMNs are shown in 2c.

For instance, with SC, since WLANs are only allowed to transmit in their primary channel, the CTMN can only transit from state $\emptyset$ to states $\mathrm{A}_{1}^{1}$ or $\mathrm{B}_{2}^{2}$, i.e., $\alpha_{\mathrm{A}, \emptyset}\left(s_{2}\right)=$ $\alpha_{\mathrm{B}, \emptyset}\left(s_{4}\right)=1$. Instead, with SCB, WLANs can only transmit in their complete allocated channel, thus, when being in state $\emptyset$ the CTMN transits to the all or nothing states $\mathrm{A}_{1}^{2}$ or $\mathrm{B}_{1}^{2}$, i.e., $\alpha_{\mathrm{A}, \emptyset}\left(s_{3}\right)=\alpha_{\mathrm{B}, \emptyset}\left(s_{5}\right)=1$. Notice that in this particular case AM generates the same transition probabilities (and respective average throughput) than SCB because whenever the WLANs have the possibility to transmit - which only happens when the CTMN is in state $\emptyset-$ they pick the widest channel available, i.e., $C_{\mathrm{A}}^{\mathrm{tx}}=C_{\mathrm{B}}^{\mathrm{tx}}=\{1,2\}$. Finally, PU picks uniformly at random any of the possible transitions when the backoff terminates in $\emptyset$, i.e., $\alpha_{\mathrm{A}, \emptyset}\left(s_{2}\right)=\alpha_{\mathrm{A}, \emptyset}\left(s_{3}\right)=1 / 2$ and $\alpha_{\mathrm{B}, \emptyset}\left(s_{4}\right)=\alpha_{\mathrm{B}, \emptyset}\left(s_{5}\right)=1 / 2$, respectively.

\section{Toy scenario I: to overlap or not?}

In Figure $2 \mathrm{~d}$ there are plotted the average of the $\rho$ metric, throughput and delay of both WLANs when operating under different policies and traffic loads. While we keep the traffic load of $A$ constant (i.e., $\ell_{\mathrm{A}}=100 \mathrm{pkt} / \mathrm{s}$ ), the load of $\mathrm{B}$ is the $\mathrm{x}$-axis independent variable (i.e., $\ell_{\mathrm{B}} \in[0,300] \mathrm{pkt} / \mathrm{s}$ ). We assume that both WLANs implement exactly the same policy in every case. The duration of a successful packet transmission $T_{\text {suc }}$ in 11ax depends on the length of the packets involved (i.e., RTS, CTS, data, ACK/BACK), and on the backoff slot and inter-frame durations (i.e., DIFS and SIFS). Essentially, the maximum capacity of a successful transmission (i.e., $r=1 / T_{\text {suc }}$ ) considered in these scenarios is $r_{20} \approx 159 \mathrm{pkt} / \mathrm{s}$ for single-channel $(20 \mathrm{MHz})$ transmissions, and $r_{40} \approx 318$ $\mathrm{pkt} / \mathrm{s}$ for two bonded channels $(40 \mathrm{MHz}$ ) transmissions. Table I shows the values of the parameters considered in the scenarios discussed throughout this work.

Regarding A's saturation point, we note that, as singlechannel capacity already copes with $\ell_{\mathrm{A}}$ (i.e., $\ell_{\mathrm{A}}<r_{20}$ ), it never gets saturated (i.e., $\rho_{\mathrm{A}}<1$ ) no matter neither the policy selected nor $\ell_{\mathrm{B}}{ }^{5}$ Instead, B gets saturated with $\mathrm{SC}$ when $\ell_{\mathrm{B}}$ approximates $r_{20}$. As expected, with AM, B gets saturated for higher $\ell_{\mathrm{B}}$ since more packets can be transmitted per unit of time. Note that in isolation, $B$ would saturate for a $\ell_{B}$ close to $r_{40}$. In this case, however, the whole channel is shared with A when both implement AM.

In terms of throughput, the higher the traffic load required to saturate a WLAN, the higher its potential value. That is,

\footnotetext{
${ }^{5}$ Note that $\ell<r$ is a mandatory condition in order to ensure unsaturated regimes. The reason lies in the overheads caused by the headers and inter frame spaces considered in the duration of a successful packet transmission.
}

TABLE I: Parameters considered in the presented scenarios.

\begin{tabular}{lll}
\hline Parameter & Description & Value \\
\hline CCA & CCA threshold & $-82 \mathrm{dBm}$ \\
$P_{\mathrm{tx}}$ & Transmission power & $15 \mathrm{dBm}$ \\
$G_{\mathrm{tx}}$ & Transmitting gain & $0 \mathrm{~dB}$ \\
$G_{\mathrm{rx}}$ & Reception gain & $0 \mathrm{~dB}$ \\
$L_{\mathrm{data}}$ & Length of a data packet & $12000 \mathrm{bits}$ \\
$L_{\mathrm{BACK}}$ & Length of a block ACK & $240 \mathrm{bits}$ \\
$L_{\mathrm{RTS}}$ & Length of an RTS packet & $160 \mathrm{bits}$ \\
$L_{\mathrm{CTS}}$ & Length of a CTS packet & $112 \mathrm{bits}$ \\
$n_{\mathrm{agg}}$ & Num. data packets aggregated & 64 \\
CE & Capture effect threshold & $20 \mathrm{~dB}$ \\
$N$ & Background noise level & $-95 \mathrm{dBm}$ \\
$T_{\mathrm{slot}}$ & Slot duration & $9 \mu \mathrm{s}$ \\
SIFS & SIFS duration & $16 \mu \mathrm{s}$ \\
DIFS & DIFS duration & $34 \mu \mathrm{s}$ \\
PIFS & PIFS duration & $25 \mu \mathrm{s}$ \\
$\eta$ & Packet error rate & 0.1 \\
$f_{c}$ & Central frequency & $5 \mathrm{GHz}$ \\
$T_{\mathrm{ofdm}}$ & OFDM symbol duration & $16 \mu \mathrm{s}$ \\
$T_{\mathrm{phy}}$ & Legacy PHY header duration & $20 \mu \mathrm{s}$ \\
$n_{\mathrm{ss}}$ & SU spatial streams & 1 \\
$T_{\mathrm{phy}}^{\mathrm{HE}}$ & HE header duration & $32 \mu \mathrm{s}$ \\
$L_{\mathrm{sf}}$ & Length of MAC's service field & $16 \mathrm{bits}$ \\
$L_{\mathrm{del}}$ & Length of MAC's MPDU delimiter & $32 \mathrm{bits}$ \\
$L_{\mathrm{mac}}$ & Length of MAC header & $272 \mathrm{bits}$ \\
$L_{\mathrm{tail}}$ & Length of MAC's tail & $6 \mathrm{bits}$ \\
PL & Path loss at distance $d$ for $5 \mathrm{GHz}$ in- & PLFree $(d)+$ \\
& door environments with corridors $[15]$ & $0.44 d$ \\
\hline & & \\
\hline & & \\
\hline
\end{tabular}

AM provides the highest $\Gamma_{\mathrm{B}}$ for high $\ell_{\mathrm{B}}$, while any policy combination copes with $\ell_{\mathrm{A}}$ (i.e., $\ell_{\mathrm{A}}=\Gamma_{\mathrm{A}}$ ). Likewise, in terms of delay, ${ }^{6}$ AM provides the best average performance to both WLANs, for every load traffic combination. Note that, once B gets saturated, the difference on the delay values between the policies is precisely the transmission time of a single packet.

In this particular scenario, an overlapping approach is the best both in terms of delay and throughput. However, in the worst case when both A and B get saturated, SC and PU outperform AM [12].

\section{Toy Scenario II: drawbacks of overlapping}

Scenario II shown in Figure 3a comprises a network of three WLANs where the central one (B) is in the carrier sense range of the other two (A and $\mathrm{C}$ ). Instead, $\mathrm{A}$ and $\mathrm{C}$ are outside the carrier sense of each other (i.e., the edge WLANs never overlap in any basic channel). We consider two different channel allocations for comparing the non-overlapping vs. overlapping approaches, respectively:

- $C_{\mathrm{no}}: C_{\mathrm{A}}=C_{\mathrm{C}}=\{1(p), 2\}$ and $C_{\mathrm{B}}=\{3(p), 4\}$.

- $C_{\mathrm{ov}}: C_{\mathrm{A}}=C_{\mathrm{C}}=\{1(p), 2,3,4\}$ and $C_{\mathrm{B}}=\{1,2,3(p), 4\}$.

Note that, as shown in Figures $3 \mathrm{~b}$ and $3 \mathrm{c}$, different states are reached depending on the channel allocation of the WLANs. On the one hand, $C_{\text {no }}$ allows any combination of concurrent transmissions by sacrificing potential allocated bandwidth. On the other hand, WLANs must content for the channel when $C_{\mathrm{ov}}$ is allocated. In turn, their data rate is approximately doubled with respect to $C_{\text {no }}$ (i.e., $r_{80} \approx 2 \cdot r_{40}$ ).

In Figure 3d, the delay experienced by the WLANs under different traffic loads is shown. Note that $\mathrm{A}$ and $\mathrm{B}$ behave

\footnotetext{
${ }^{6}$ Delay is estimated using the 11 axHDWLANSim wireless networks simulator.
} 


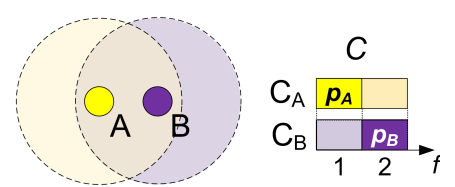

(a)

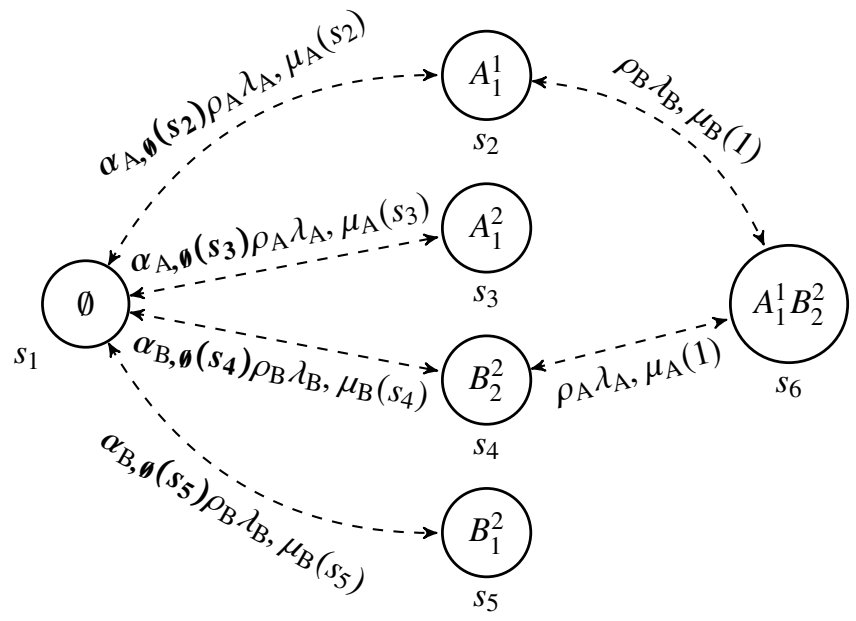

(c)

\begin{tabular}{cccccc}
\hline $\mathcal{D}$ & $|\mathcal{S}|$ & $\overrightarrow{\boldsymbol{\alpha}}_{\mathrm{A}, \emptyset}\left(s_{\mathbf{2}}\right)$ & $\overrightarrow{\boldsymbol{\alpha}}_{\mathrm{A}, \emptyset}\left(s_{\mathbf{3}}\right)$ & $\overrightarrow{\boldsymbol{\alpha}}_{\mathrm{B}, \emptyset}\left(s_{\mathbf{4}}\right)$ & $\overrightarrow{\boldsymbol{\alpha}}_{\mathrm{B}, \emptyset}\left(s_{\mathbf{5}}\right)$ \\
\hline $\mathrm{SC}$ & 4 & 1.0 & 0.0 & 1.0 & 0.0 \\
$\mathrm{SCB}$ & 3 & 0.0 & 1.0 & 0.0 & 1.0 \\
$\mathrm{AM}$ & 3 & 0.0 & 1.0 & 0.0 & 1.0 \\
$\mathrm{PU}$ & 6 & 0.5 & 0.5 & 0.5 & 0.5 \\
\hline
\end{tabular}

(b)

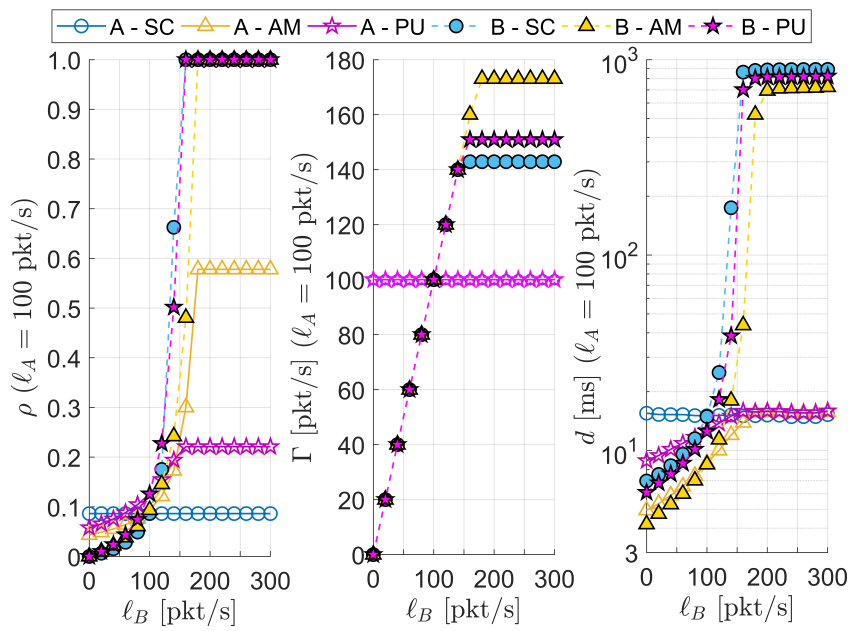

(d)

Fig. 2: Scenario I. (a) WLANs A and B are inside the carrier sense range of each other with potentially overlapping basic channels 1 and 2. (b) Number of feasible states and transition probabilities of WLANs A and B in Scenario I for different DCB policies. (c) The states and transitions of the CTMN depend on the applied DCB policies. (d) Average $\rho$ metric, throughput and delay experienced by WLANs A and $B$ under different DCB policies and traffic loads.

exactly the same. We evaluate the delay for three different values of $\ell_{\mathrm{B}}$ (i.e., 100,250 and $400 \mathrm{pkt} / \mathrm{s}$ ) and a range $\ell_{A}=\ell_{C}=\ell_{e} \in[0,600] \mathrm{pkt} / \mathrm{s}$. As expected, for the nonoverlapping case, there is no dependence among WLANs. That is, their delay is just consequence of its own traffic load, and get saturated only when this approximates $r_{40}$.

Regarding the overlapping channel allocation, results show that, for low traffics, AM is the best policy for the three WLANs. Nonetheless, for higher $\ell_{\mathrm{e}}$, B's performance is really deteriorated. Essentially, while $\mathrm{A}$ and $\mathrm{C}$ can transmit at the same time whenever B is not active, B can only do so when neither A nor $\mathrm{C}$ are active. This is a clear case of unfair WLAN starvation. Namely, the larger $\ell_{e}$, the fewer the transmission opportunities for $\mathrm{B}$, as $\mathrm{A}$ and $\mathrm{C}$ transmit during the majority of time. Interestingly, $\ell_{\mathrm{B}}$ does not practically affect to $d_{\mathrm{A}}$ or $d_{\mathrm{C}}$, since B starves even for $l_{\mathrm{B}}=100 \mathrm{pkt} / \mathrm{s}$ when $\ell_{e}$ is high.

Then, we see that, while an overlapping approach is really convenient for the edge WLANs, it is not the case for WLAN $B$ when the edge traffic load is high, since B actually starves. Nonetheless, for low aggregated network traffic loads $\left(\ell_{\mathrm{e}}+\ell_{\mathrm{B}}\right)$, $d_{\mathrm{B}}$ is also improved in the overlapping approach. Note that the later case is the most likely to happen since real WLANs operate on average under low traffic loads.

The presented toy scenarios allow us to notice that there is not a unique spectrum management approach that suits all the cases. In fact, WLANs performance depend on multiple parameters like spatial distribution and traffic loads, but also on the metric objective to be optimized, which may be designed to foster individual or collaborative behaviors. Nonetheless, we have seen that, as a rule of thumb, AM and overlapping channel allocations are convenient for low to moderate traffic loads.

\section{Performance eValuation in Dense scenarios}

In this Section we discuss what is the approach that a particular WLAN should locally implement for maximizing its own performance. The results gathered have been obtained using 11 axHDWLANS im, ${ }^{7}$ an event-based wireless network simulator that implements new technologies included in the IEEE 802.11ax amendment.

\section{A. Scenario under evaluation}

As shown in Figure 4a, we consider a 50 x $50 \mathrm{~m}^{2}$ map with one WLAN (A) located at the center, and $M-1=19$ WLANs spread uniformly at random in the area with the single condition that any pair of APs must be separated at least $d_{\mathrm{AP}-\mathrm{AP}}^{\min }=10$ $\mathrm{m}$. The $\mathrm{STA}^{8}$ of each WLAN is located also uniformly at random at a distance $d_{\mathrm{AP}-\mathrm{STA}} \in\left[d_{\mathrm{AP}-\mathrm{STA}}^{\min }, d_{\mathrm{AP}-\mathrm{STA}}^{\max }\right]=[1,5] \mathrm{m}$ from the AP. Regarding the channel allocation, all the WLANs

\footnotetext{
${ }^{7}$ All of the source code of Komondor is open, encouraging sharing of algorithms between contributors and providing the ability for people to improve on the work of others under the GNU General Public License v3.0. The code used in this work can be found at https://github.com/wn-upf/ Komondor/releases/tag/v1.2.

${ }^{8}$ Due to the fact that APs and STAs are located randomly in the map, the number of STAs should not have a significant impact on the results because only downlink traffic is assumed.
} 


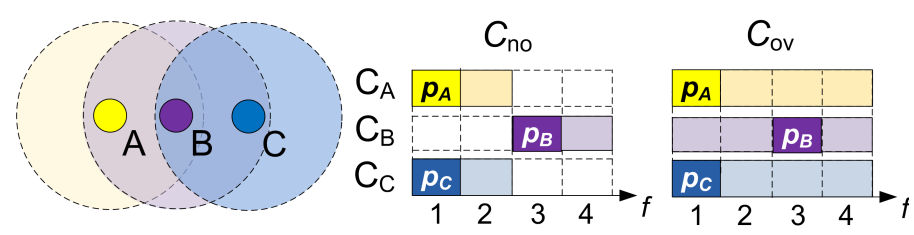

(a)

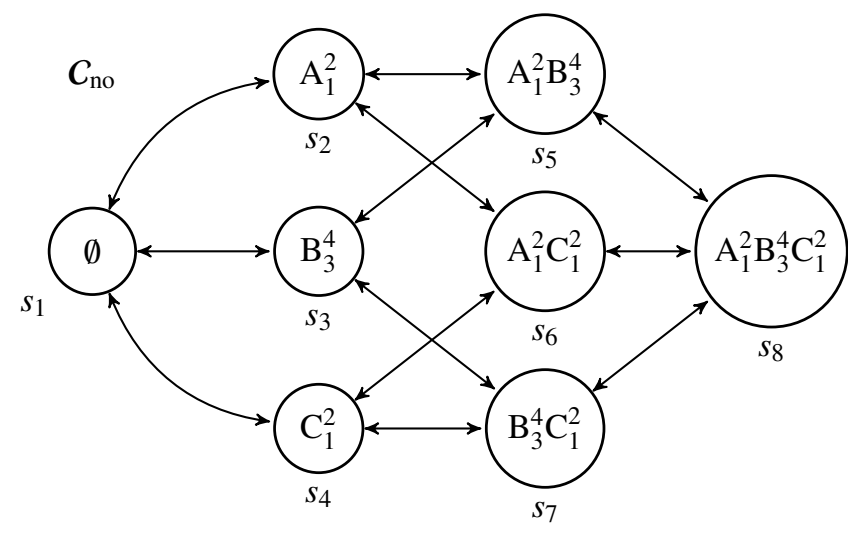

(b)
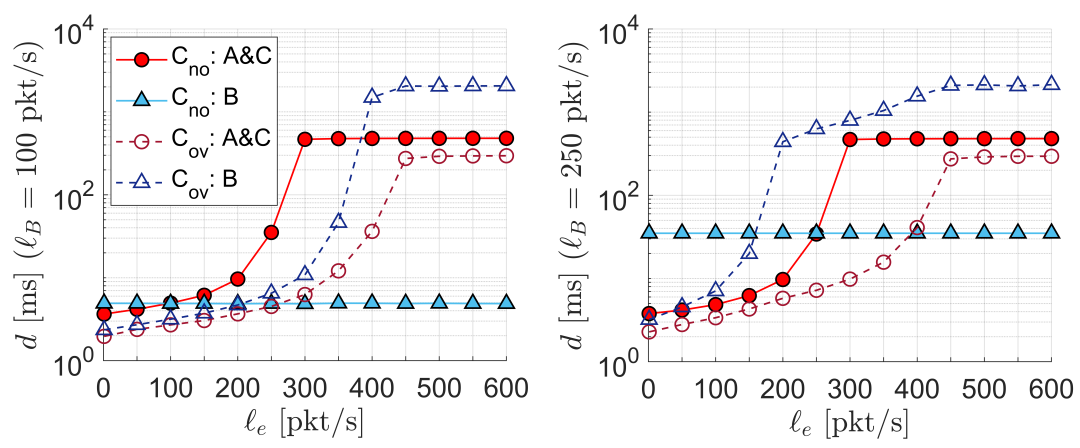

(d)

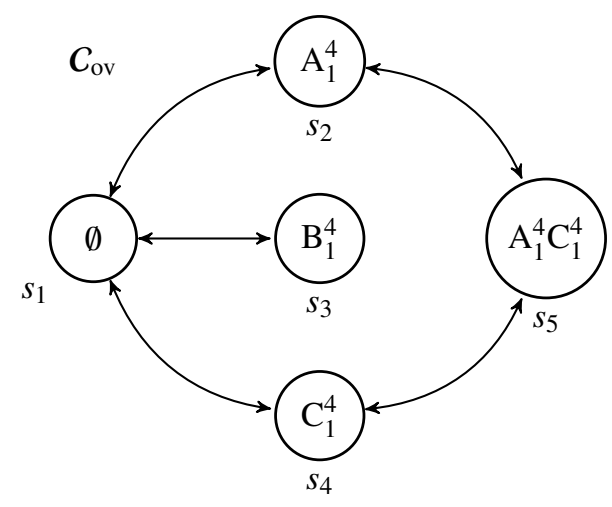

(c)

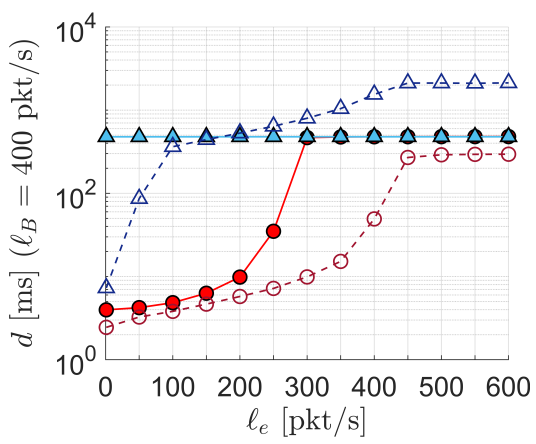

Fig. 3: Scenario II: AM on a spatial distributed network. (a) A neighbor overlapping network where two channel allocations are considered. (b) CTMN corresponding to the non-overlapping channel allocation $C_{\mathrm{no}}$. (c) CTMN corresponding to the overlapping channel allocation $C_{\mathrm{ov}}$. (d) Traffic load impact on the average delay experienced by the WLANs.

are set with random primary channels in the eight basic channels considered in the system (i.e., $p_{\mathrm{w}} \sim U[1,8], \forall w$ ). The set of allocated basic channels is assigned uniformly at random as well. That is, the number of allowed basic channels for transmitting is $\left|C_{w}\right| \sim U\{1,2,4,8\}, \forall w$, with the exception of WLAN A, which is allocated the widest channel (i.e., $\left.C_{\mathrm{A}}=\{1, \ldots, 8\}\right)$. Besides, we consider bursty traffic dependent on the average traffic load $(\ell)$, where a burst of $n_{b}=10$ packets is generated each $t_{b} \sim \operatorname{Exponential}\left(n_{b} / \ell\right)$ in order to provide more realistic traffic patterns.

While the DCB policies of the $M-1$ WLANs are also set uniformly at random (i.e., they implement SC, SCB, AM or PU with same probability $1 / 4$ ), A is fixed to a desired policy. Specifically, we generate $N_{\mathrm{D}}=300$ deployments following the aforementioned conditions for each of the $N_{\mathrm{P}}=4$ policies that A can implement. Besides, we evaluate each policy for $N_{\ell}=13$ values of A's traffic load $\left(\ell_{\mathrm{A}}=1,20,40, \ldots, 240\right)$. The rest of WLANs are set with random average traffic load $\ell_{w} \sim U[1,240]$. Hence, we simulate $N_{\mathrm{D}} \times N_{\mathrm{P}} \times N_{\ell}=15600$ scenarios. The simulation time of each scenario is 25 seconds. In order to compute the delay corresponding to the stationary regime, we average the delay per packet only during the last 5 seconds of every simulation.

\section{B. Results}

Figure $4 \mathrm{~b}$ shows the probability of WLAN A to successfully transmit all its traffic load. That is, with probability $P_{\mathrm{A}}=$ $\mathcal{P}\left(\Gamma_{\mathrm{A}} \geq\left(1-\epsilon_{\Gamma}\right) \ell_{\mathrm{A}}\right)$ A does not get saturated. Note that we use a margin of error $\epsilon_{\Gamma}=0.05$ to cope with the stochastic packet generation of the performed simulations. As expected, SCB is viable only for few scenarios when $\ell_{\mathrm{A}}=1 \mathrm{pkt} / \mathrm{s}$ because the rest of WLANs most likely prevent A to initiate transmissions by occupying part of its allocated channel $C_{\mathrm{A}}$. Instead, the other policies perform much better since they avoid saturation with high probability even for high traffic loads; specially AM. While $\mathrm{A}$ avoids saturation in some scenarios for $\ell_{\mathrm{A}}<160$ 


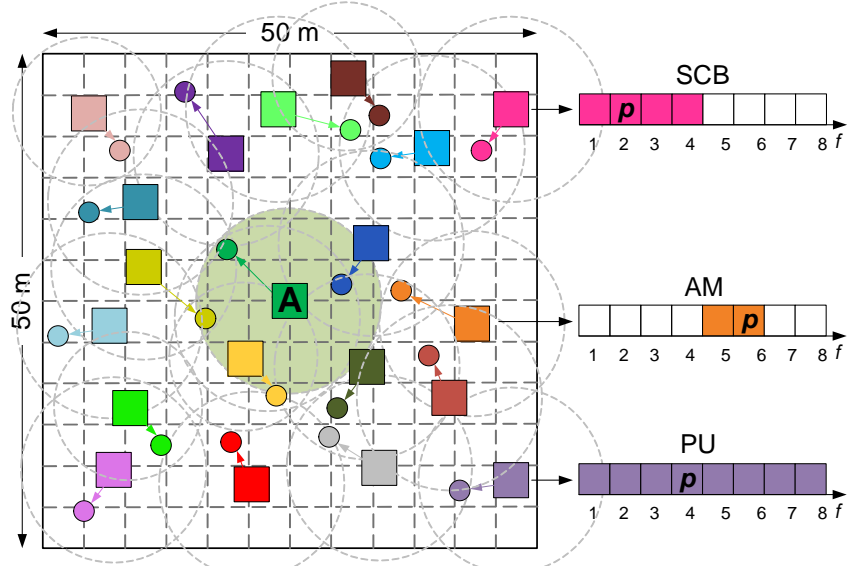

(a)

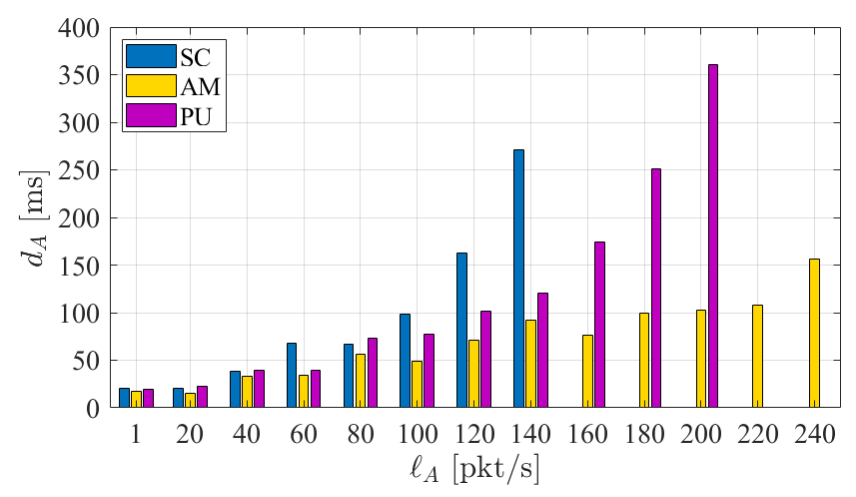

(c)

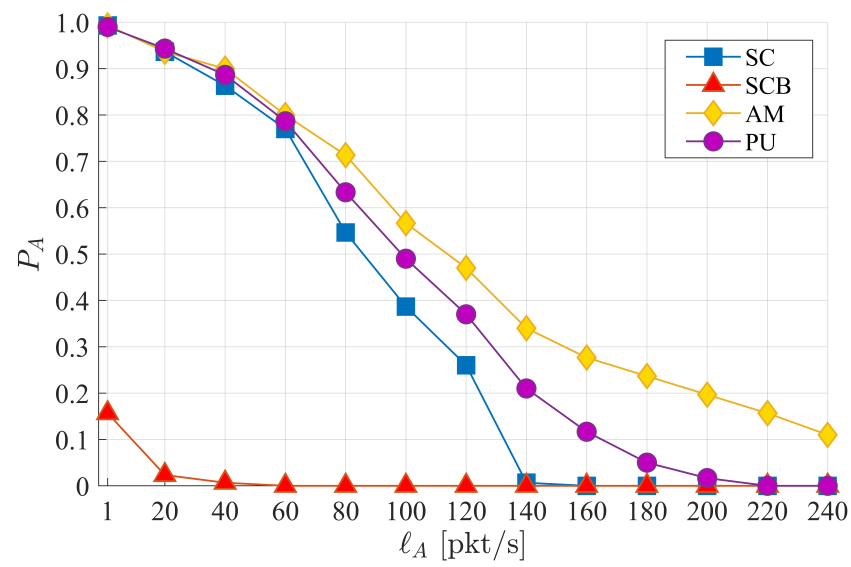

(b)

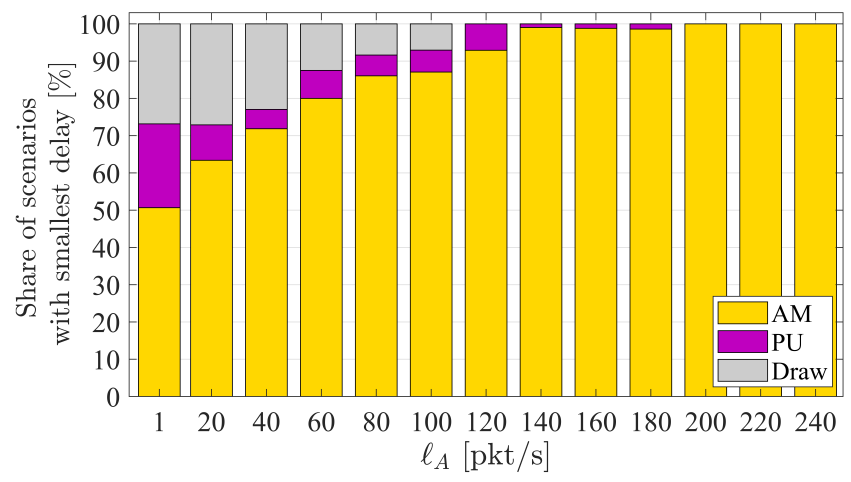

(d)

Fig. 4: Central WLAN scenario. (a) Random deployment with $M=20$. (b) Traffic load effect on the probability that A achieves a throughput similar to its traffic load. (c) Average delay of A in non-saturated scenarios. (d) Share of scenarios where AM or PU provide the smallest delay for A in non-saturated scenarios.

$\mathrm{pkt} / \mathrm{s}$ with $\mathrm{SC}$ and $\ell_{\mathrm{A}}<220 \mathrm{pkt} / \mathrm{s}$ with $\mathrm{PU}$, respectively, the aggressive adaptability nature of AM makes it able to avoid saturation even for some scenarios where $\ell_{A}=240 \mathrm{pkt} / \mathrm{s}$.

The average delay experienced by A under different traffic loads is shown in Figure 4c. We consider only those scenarios when A does not get saturated (see Figure 4b) in order to fairly compare among the presented policies. As a significant result, we note that the smallest average delay is provided by AM for all the studied loads. However, there are particular scenarios where a less aggressive approach like PU outperforms AM. In this regard, Figure $4 \mathrm{~d}$ shows the share of scenarios where $\mathrm{AM}$ or PU are the policies providing the smallest $d_{\mathrm{A}}$. Three types of outcomes are categorized according to a defined delay margin $\delta_{d}=1 \mathrm{~ms}$ for capturing the cases where $\mathrm{AM}$ and $\mathrm{PU}$ perform similarly:

$$
\text { if } \mathrm{E}\left[d_{\mathrm{A}}^{\mathrm{PU}}\right]-\mathrm{E}\left[d_{\mathrm{A}}^{\mathrm{AM}}\right] \begin{cases}<-\delta_{d}, & \mathrm{PU} \text { better than } \mathrm{AM} \\ >\delta_{d}, & \mathrm{PU} \text { worse than } \mathrm{AM} \\ \text { otherwise, } & \text { PU similar to } \mathrm{AM}\end{cases}
$$

We see that in most of the cases AM outperforms PU, specially for scenarios with high traffic loads. Nonetheless, there are scenarios with lower loads where PU provides smaller delays than AM. This mainly occurs when A and its neighboring nodes are able to concurrently transmit in different channels through interactions that are not given when implementing AM. Essentially, when A transmits in its whole available bandwidth, neighboring WLANs with primary channels overlapping with A's transmission must wait until it is finished. Afterwards, such WLANs are able to terminate their backoffs and could select a transmission channel including A's primary in turn. This generates all or nothing states like the one shown in Scenario I that keep A's backoff frozen for longer periods of time. Instead, if A transmits in narrower channels by implementing PU, such WLANs could transmit at the same time in non-overlapping channels and enable more successful parallel transmissions.

In summary, we see that overlapping approaches can significantly enhance traditional single-channel performance in terms of delay and throughput. Still, there are cases when an overlapping approach that always selects the maximum available bandwidth is counterproductive in the mid/long-term. Besides, fairness issues must be taken into consideration too when aggressive DCB is implemented. Despite the intrinsic uncertainty of spatially distributed WLAN deployments, we can state as a rule of thumb that DCB is convenient when applied through spectrum-adapting policies. Nonetheless, as 
indicated by the scenarios where PU outperformed AM, there is room for further improvement through smarter adaptation by taking advantage of the potential knowledge gathered, and adopting policies on a per-WLAN basis.

\section{Conclusions}

In this work we assess the performance of channel bonding in WLANs under variable traffic loads. By modeling and simulating DCB policies in spatially distributed scenarios we shed light on the question: is it convenient to share wider channels and overlap in spectrum or not? We show that, while the performance of static channel bonding is clearly poor, spectrum-adapting DCB significantly outperforms the traditional single-channel approach for low to moderate traffic loads, even in high density deployments.

Indeed, this suggests that future WLANs should be allocated all the available spectrum, and be able to locally adopt proper DCB policies, which contradicts most of current thoughts, that push towards non-overlapping channels. Still, for high traffic loads, fairness issues like starvation may appear as a consequence of the spatial distribution effects. Accordingly, less aggressive DCB policies like stochastic width selection may perform better. In this regard, the intricate nature of spatially distributed WLAN environments leaves room for further improvement through intelligent DCB approaches.

\section{ACKNOWLEDGMENT}

This work has been partially supported by the Spanish Ministry of Economy and Competitiveness under the Maria de Maeztu Units of Excellence Programme (MDM-20150502), and by a Gift from the Cisco University Research Program (CG\#890107, Towards Deterministic Channel Access in High-Density WLANs) Fund, a corporate advised fund of Silicon Valley Community Foundation. The work done by S. Barrachina-Muñoz is supported by a FI grant from the Generalitat de Catalunya.

\section{REFERENCES}

[1] Mohammed S Elbamby, Cristina Perfecto, Mehdi Bennis, and Klaus Doppler. Towards low-latency and ultra-reliable virtual reality. arXiv preprint arXiv:1801.07587, 2018.

[2] Lara Deek, Eduard Garcia-Villegas, Elizabeth Belding, Sung-Ju Lee, and Kevin Almeroth. The impact of channel bonding on $802.11 \mathrm{n}$ network management. In Proceedings of the Seventh COnference on emerging Networking EXperiments and Technologies, page 11. ACM, 2011.

[3] IEEE 802.11n. Standard for Wireless LAN Medium Access Control (MAC) and Physical Layer (PHY): Enhancements for High Throughput. IEEE, 2009.

[4] I. P802.11ac. Standard for Wireless LAN Medium Access Control (MAC) and Physical Layer (PHY) specifications: Enhancements for Very High Throughput for Operation in Bands below $6 \mathrm{GHz}$. IEEE, 2014.

[5] B. Bellalta. IEEE 802.11ax: High-efficiency WLANs. IEEE Wireless Communications, 23(1):38-46, 2016.

[6] Syed Hashim Raza Bukhari, Mubashir Husain Rehmani, and Sajid Siraj. A survey of channel bonding for wireless networks and guidelines of channel bonding for futuristic cognitive radio sensor networks. IEEE Communications Surveys \& Tutorials, 18(2):924-948, 2016.

[7] IEEE 802.11 Task Group AX. Status of Project IEEE 802.11ax High Efficiency WLAN (HEW). http://www.ieee802.org/11/Reports/tgax update.htm. Accessed: 2018-03-21.

[8] Minyoung Park. IEEE 802.11 ac: Dynamic bandwidth channel access. In Communications (ICC), 2011 IEEE International Conference on, pages 1-5. IEEE, 2011.
[9] Boris Bellalta, Azadeh Faridi, Jaume Barcelo, Alessandro Checco, and Periklis Chatzimisios. Channel bonding in short-range WLANs. In $E u-$ ropean Wireless 2014; 20th European Wireless Conference; Proceedings of, pages 1-7. VDE, 2014.

[10] Rafael Laufer and Leonard Kleinrock. On the capacity of wireless CSMA/CA multihop networks. In INFOCOM, 2013 Proceedings IEEE, pages 1312-1320. IEEE, 2013.

[11] Boris Bellalta, Alessandro Checco, Alessandro Zocca, and Jaume Barcelo. On the interactions between multiple overlapping WLANs using channel bonding. IEEE Transactions on Vehicular Technology, 65(2):796-812, 2016.

[12] Sergio Barrachina-Muñoz, Francesc Wilhelmi, and Boris Bellalta. Performance Analysis of Dynamic Channel Bonding in Spatially Distributed High Density WLANs. arXiv preprint arXiv:1801.00594, 2018.

[13] Azadeh Faridi, Boris Bellalta, and Alessandro Checco. Analysis of Dynamic Channel Bonding in Dense Networks of WLANs. IEEE Transactions on Mobile Computing, 2016.

[14] Periklis Chatzimisios, Anthony C Boucouvalas, and Vasileios Vitsas. Performance analysis of IEEE 802.11 DCF in presence of transmission errors. In Communications, 2004 IEEE International Conference on, volume 7, pages 3854-3858. IEEE, 2004.

[15] Jonas Medbo and J-E Berg. Simple and accurate path loss modeling at $5 \mathrm{GHz}$ in indoor environments with corridors. In Vehicular Technology Conference, 2000. IEEE-VTS Fall VTC 2000. 52nd, volume 1, pages 30-36. IEEE, 2000.

\section{BIOGRAPHIES}

SERGIO BARRACHINA-MUÑOZ obtained his B.Sc. degree in Telematics Engineering and his M.Sc. in Intelligent Interactive Systems in 2015 and 2016, respectively, both from Universitat Pompeu Fabra (UPF), Barcelona. Currently, he is a $\mathrm{PhD}$ student and teacher assistant in the Wireless Networking (WN) research group at Universitat Pompeu Fabra (UPF). His main research interests are focused on developing autonomous learning methods and techniques for improving the performance of next-generation wireless networks.

FRANCESC WILHELMI holds a BSc degree in Telematics Engineering from the Universitat Pompeu Fabra (2015) with a focus on Broadband and Wireless Communications. With the aim of applying new techniques for solving many well-known problems in communications, Francesc obtained his MSc degree in Intelligent and Interactive Systems also from the UPF in 2016. He is now a PhD Student in the Wireless Networking Group (WN) of the Department of Information and Communication Technologies (DTIC) at the UPF. The main topics of his $\mathrm{PhD}$ Thesis are related to spatial reuse in high-density wireless networks through power and sensitivity adjustment by taking advantage of Reinforcement Learning (RL) techniques.

BORIS BELLALTA is an Associate Professor in the Department of Information and Communication Technologies (DTIC) at Universitat Pompeu Fabra (UPF). He obtained his degree in Telecommunications Engineering from Universitat Politècnica de Catalunya (UPC) in 2002 and the Ph.D. in Information and Communication Technologies from UPF in 2007. His research interests are in the area of wireless networks, with emphasis on the design and performance evaluation of new architectures and protocols. The results from his research have been published in more than 100 international journal and conference papers. He is currently involved in several international and national research projects, including the coordination of the ENTOMATIC FP7 collaborative project. At UPF he is giving several courses on networking, queuing theory and wireless networks. He is co-designer and coordinator of the interuniversity (UPF and UPC) masters degree in Wireless Communications. 